\title{
The Short-Form Inguinal Pain Questionnaire (sf-IPQ): An Instrument for Rating Groin Pain After Inguinal Hernia Surgery in Daily Clinical Practice
}

\author{
Anders Olsson $^{1,2}$ - G. Sandblom ${ }^{1,2}$ • U. Fränneby ${ }^{1,3} \cdot$ A. Sondén ${ }^{1,2} \cdot$ U. Gunnarsson ${ }^{4}$. \\ U. Dahlstrand ${ }^{3,5}$
}

Published online: 27 November 2018

(C) The Author(s) 2018

\begin{abstract}
Background The Inguinal Pain Questionnaire (IPQ) is a standardised and validated instrument for assessing persisting pain after groin hernia surgery. The IPQ is often perceived as being too extensive for routine use. The aim of this study was to develop and evaluate a condensed version of the IPQ in order to facilitate its use in daily clinical practice.

Methods The condensed form, i.e. Short-Form Inguinal Pain Questionnaire (sf-IPQ), comprises two main items taken from the IPQ. Four hundred patients were recruited from the Swedish Hernia Register and were sent the IPQ, sf-IPQ and the Short-Form McGill Pain Questionnaire (SF-MPQ) three years after hernia repair. Ratings from the IPQ and the sf-IPQ were converted to a 12-point scale. The reported scores for the two shared items in the IPQ and sfIPQ were compared using the Intraclass Correlation Coefficient (ICC), Cohen's kappa and McNemar's test.

Results After two reminders, the response rate was $69.8 \%(n=279 / 400)$. The ICC for the IPQ and sf-IPQ scores was 0.78 (95\% confidence interval $0.73-0.82, p<0.001$ ). Cohen's kappa was 0.66 (95\% confidence interval $0.55-0.77$, $p<0.001)$. The sf-IPQ systematically indicated a higher pain score than the IPQ $(p=0.013)$.

Conclusions Despite the systematic difference in level of pain scored, correlation, consistency and agreement were seen between the IPQ and sf-IPQ. The forms appear to be interchangeable, though the sf-IPQ may be a more sensitive instrument. The condensed structure of the sf-IPQ is more user-friendly and shows promise as a useful tool in daily clinical practice.
\end{abstract}

Anders Olsson

anders.olsson@ki.se

1 Department of Clinical Science and Education, Karolinska Institutet, Södersjukhuset, Stockholm, Sweden

2 Department of Surgery, Södersjukhuset, Sjukhusbacken 10, 11883 Stockholm, Sweden

3 Center for Digestive Diseases, Karolinska University Hospital, Stockholm, Sweden

4 Division of Surgery, Department of Surgical and Perioperative Sciences, Umeå University, Umeå, Sweden

5 Department of Clinical Sciences, Intervention and Technology (CLINTEC), Karolinska Institutet, Stockholm, Sweden

\section{Introduction}

Persisting pain is common after inguinal hernia repair $[1,2]$ and considered to be one of the most important outcome measures after hernia surgery today. In its most severe form, persisting pain may cause disability and necessitate reoperation [3]. The International Association for the Study of Pain (IASP) Task Force for the ICD classification of post-operative chronic pain defined it as pain lasting 3 months or more after trauma or surgery, where persisting infectious or pre-existing conditions have been ruled out as being the cause [4]. Pain, however, is a subjective experience and therefore difficult to characterise and measure. Pain is defined by the IASP as "An 
unpleasant sensory and emotional experience associated with actual or potential tissue damage or described in terms of such damage".

Several instruments have been developed to measure different aspects of pain, e.g. the McGill Pain Questionnaire which is one of the most used instruments for evaluating general pain [5]. Various studies have reported a $10-30 \%$ prevalence of persisting pain following hernia repair depending on the criteria used [6-8]. This divergence in rates may be explained by the lack of a uniformly accepted definition of persisting pain and the use of different methods of assessment. Young age, pain prior to surgery and severe early post-operative pain are known risk factors for persisting pain following hernia repair [8,9].

Standardised criteria for the evaluation of persisting pain are needed if we are to effectively study strategies aiming to avoid this problem. Groin hernia surgery is one of the most frequently performed procedures in general surgery; thus, the number of patients that are at risk is substantial. Patient-reported post-operative pain (short as well as long term) is an important measure of quality in inguinal hernia repair. The disease-specific Inguinal Pain Questionnaire (IPQ) was developed to establish a standardised instrument for rating inguinal pain after groin hernia surgery [10]. The original IPQ was designed to specifically assess preoperative as well as post-operative inguinal pain and has been validated against the Brief Pain Inventory, a pain questionnaire developed for evaluating general pain in cancer patients [11]. The IPQ consists of 18 items covering pain intensity and interference with daily activities [8]. It is a well-cited instrument but is considered by many to be too comprehensive for use in daily clinical practice. A condensed version with fewer items could facilitate its use in clinical practice.

The aim of this study was to see whether a shortened form of the IPQ, having the benefit of brevity, provides outcome values that correspond to those from the IPQ.

\section{Material and method}

\section{Patients and study design}

The study was a cross-sectional analysis of a cohort. Four hundred consecutive patients who had undergone inguinal hernia repair during March 2013 were recruited from the Swedish Hernia Register (SHR), a population-based national register started in 1992 . The SHR contains data on all inguinal hernia repairs performed on patients aged 15 years and older and covers more than $95 \%$ of all groin hernia repairs in Sweden. The SHR includes information about method of repair, anaesthesia, anatomical circumstances, the surgeon and the patient. The SHR also includes information on post-operative adverse events [12]. During the spring of 2016, the recruited patients were sent an envelope containing three pain questionnaires (IPQ, sf-IPQ and the Short-Form McGill Pain Questionnaire, SF-MPQ), information about the study and a form for written consent. As 3 years had passed since hernia repair, the healing process was assumed not to have any impact on the prevalence of persisting pain. Nor should the level of pain reported have been affected by whether the patient answered the questionnaires straight away or delayed answering until after one of the reminders. In order to avoid the possibility that presenting the forms in a fixed order could affect the way the patients answered, half of the patients received the questionnaires in the order "IPQ, sf-IPQ and SF-MPQ", while the other half received the questionnaires in the order "sf-IPQ, IPQ and SF-MPQ".

\section{The sf-IPQ}

In the original IPQ, pain intensity is assessed using a 7-step fixed rate scale with steps operationally linked to pain behaviour or pain descriptors [8, 10]. The IPQ has four pages and takes approximately $10 \mathrm{~min}$ to complete [10]. The design of the sf-IPQ was based on experience gained from the wide use of the IPQ, which is considered a reliable yet somewhat too detailed questionnaire. After review of several studies where the IPQ was used, two items emerged as being most clinically important when evaluating inguinal pain. These items were taken from the IPQ to form the sf-IPQ (see Fig. 1).

Item 1 in the sf-IPQ was taken in its original form from the IPQ, whereas Item 2 was compiled from six separate

\footnotetext{
1. Estimate the worst pain you felt in the operated groin during this past week

No pain

Pain present but can easily be ignored

Pain present, cannot be ignored, but does not interfere with daily activities

Pain present, cannot be ignored, interferes with concentration on chores and daily activities

Pain present, cannot be ignored, interferes with most activities

Pain present, cannot be ignored, necessitates bed rest

Pain present, cannot be ignored, prompt medical advice sought

2. If you have experienced groin pain, to what extent has it limited your ability to perform following activities? More than one option may be selected.

Getting up from a low chair

Sitting down (more than 30 minutes)

Standing up (more than 30 minutes)

Going up or down stairs

Driving a car

Exercising or performing sports

Fig. 1 English translation of the short-form IPQ (sf-IPQ), consisting of two items with a total score ranging between 0 and 12 . Question 1 contributes 0-6 points (higher points for more intense pain) and question 2 adds one point for each reported activity limited by pain ( 6 in all)
} 
items in the IPQ regarding how groin pain interferes with specific daily activities. These two items cover one page and take less than a minute to complete.

To quantify the severity of pain, we used a scoring system originally designed for the IPQ (11), where the reported "worst level of pain during the past week" (Item 1 in the sf-IPQ) ranges from 0 points (no pain) to 6 points (pain so severe that prompt medical advice was sought). Interference with daily activities (Item 2 in the sf-IPQ) scores one point for each activity reported to be limited by groin pain. The total score thus ranges from 0 to 12 points. Scores were calculated for IPQ and sf-IPQ responders, respectively. A score of 0-2 was considered negligible pain, whereas a score of 3-12 was considered substantial pain.

\section{The McGill Pain Questionnaire}

The McGill Pain Questionnaire (MPQ) was developed in 1971 and is a well-established self-report questionnaire for rating the quality and intensity of perceived general pain [5]. It has been validated and used in a number of studies. In 1987, a short form of the MPQ was developed [13]. The short form was translated into Swedish and validated in 1994 [14]. In this study the SF-MPQ was used as a reference tool to compare the distribution of pain registered with sf-IPQ with that of the SF-MPQ using a population distribution diagram, to demonstrate whether the sf-IPQ registers the relevant modality, pain.

\section{Statistical analyses}

Based on $80 \%$ power, 95\% significance level and $15 \%$ dropout, 322 participants were required. Returned questionnaires were considered complete and included in the study if all items used to calculate the IPQ score and sf-IPQ score were answered.

IPQ score and sf-IPQ scores were compared using three methods of analysis. These statistical methods were chosen to analyse correlation between the two instruments from three points of view: Cohen's kappa was used to determine agreement regarding frequency of substantial pain; the intraclass correlation coefficient (ICC) with the two-way mixed effects model [type $\operatorname{ICC}(3,1)]$ according to Shrout and Fleiss was used as a measure of correlation and consistency of the pain score generated by the two questionnaires [15]; and the McNemar's test was used to evaluate any systematic difference between the two tests in classifying pain as substantial or negligible. Responders/nonresponders and the two questionnaire-order groups were compared with respect to age, gender, surgical technique, method of anaesthesia, emergency repair and bilateral repair. Differences were analysed using Mann-Whitney
$U$ test and Pearson's Chi-square test. Statistical analyses were performed using Stata 12.1 (StataCorp, College Station, TX, USA). A population distribution diagram was used to visualise the distribution of the sf-MPQ score in patients with sf-IPQ scores $<3$ and $\geq 3$, respectively. This diagrammatic comparison gives a picture of whether the perception of pain differs between the two instruments.

Ethics approval for the study was granted by the Regional Ethics Review Board in Stockholm (No. 2009/4431/3).

\section{Results}

The inclusion rate after two reminders and exclusion due to incomplete questionnaire was $69.8 \%$ (279/400 patients). The selection process for the study group is presented in Fig. 2. Median age of responders was 64 years, (range 23-94 years). $92.5 \% \quad(n=258)$ were men and $7.5 \%$ $(n=21)$ women. $96.4 \%(n=269)$ of the hernia repairs were planned and $3.4 \%(n=10)$ were acute. An open repair technique was used in $75.6 \%(n=211)$, while an endoscopic technique was used in $24.4 \%(n=68)$ of the cases. Two of the endoscopic repairs were converted to open repair. General anaesthesia was used in $80.3 \%$ $(n=224)$, local anaesthesia in $13.3 \%(n=37)$ and spinal/ epidural anaesthesia in $6.4 \%(n=18)$. Bilateral hernia repair was performed in $7.2 \%(n=20)$. Characteristics did not differ between responders and non-responders except for median age which was 64 years (range 23-94) for responders and 55 years (range 18-90) in the non-responder group, $p<0.001$ (Table 1). There was no difference in characteristics between the questionnaire order groups.

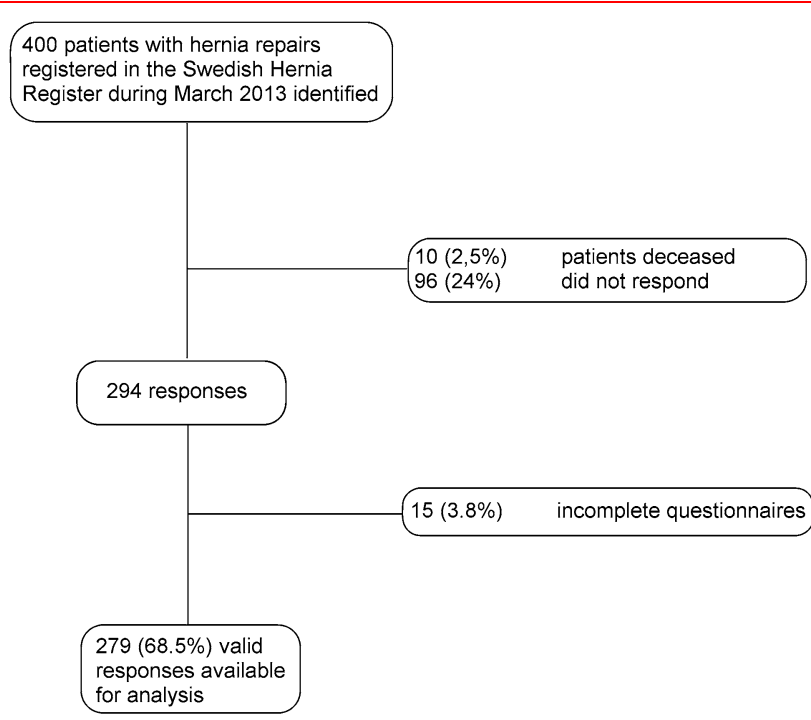

Fig. 2 Flowchart of the process of participant inclusion 
Table 1 Characteristics of responders and non-responders among the 400 patients who were sent the IPQ, sf-IPQ and SF-MPQ 3 years after inguinal hernia surgery

\begin{tabular}{|c|c|c|c|}
\hline & Responders $(N=279)$ & Non-responders $(N=121)$ & $p$ value \\
\hline Age, median (range) & $64(23-94)$ & $55(18-90)$ & $<0.001 *$ \\
\hline Gender, $n(\%)$ & & & $0.75^{\dagger}$ \\
\hline Female & $21(7.5)$ & $8(6.6)$ & \\
\hline Male & $258(92.5)$ & $113(93.4)$ & \\
\hline Surgical approach & & & $0.95^{\dagger}$ \\
\hline Open & $213(76.3)$ & $94(77.7)$ & \\
\hline Endoscopic & $66(23.7)$ & $27(22.3)$ & \\
\hline Anaesthetic & & & $0.86^{\dagger}$ \\
\hline Technique & $224(80.3)$ & $100(82.6)$ & \\
\hline General & $18(6.4)$ & $7(5.8)$ & \\
\hline Regional local & $37(13.3)$ & $14(11.6)$ & \\
\hline Mode of admission & & & $0.79^{\dagger}$ \\
\hline Elective & $269(96.4)$ & $116(95.9)$ & \\
\hline Acute & $10(3.6)$ & $5(4.1)$ & \\
\hline Uni-/bilateral & & & $0.92^{\dagger}$ \\
\hline Unilateral repair & $259(92.3)$ & $112(92.6)$ & \\
\hline Bilateral repair & $20(7.7)$ & $9(7.4)$ & \\
\hline
\end{tabular}

*Mann-Whitney $U$ test

†Pearson's Chi-squared test

IPQ score and sf-IPQ scores were calculated for all patients. The median score for both IPQ and sf-IPQ was 0 (interquartile range, IQR, $0-1$ for both instruments). The distribution of the scores is shown in Fig. 3. The frequency of patients reporting substantial pain (score $\geq 3$ ) was $7.9 \%$ $(n=22)$ for IPQ and $11.8 \%(n=33)$ for sf-IPQ. The observed agreement between IPQ and sf-IPQ regarding pain interfering with daily activities was $93.9 \%$. Cohen's kappa was 0.66 (95\% confidence interval $0.55-0.77$,

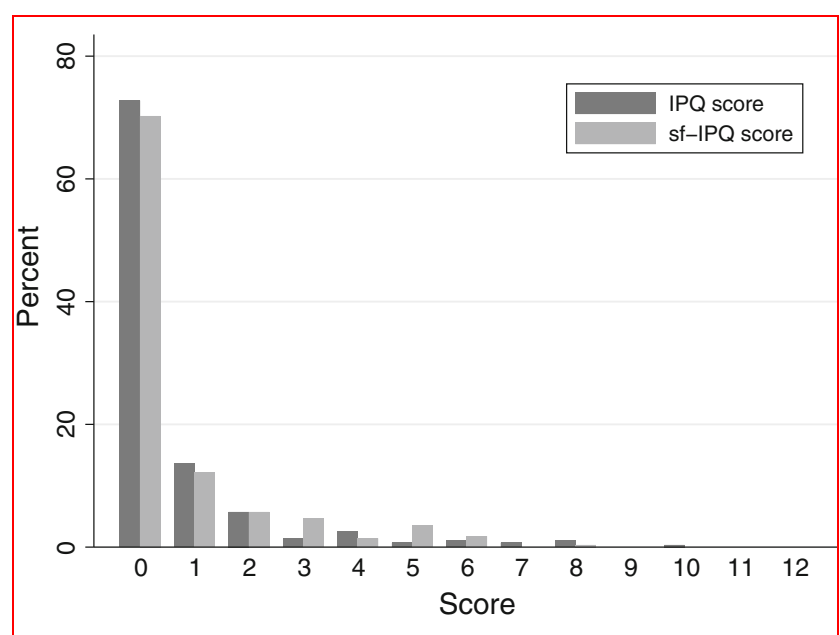

Fig. 3 Distribution of IPQ and sf-IPQ scores in 279 patients 3 years after inguinal hernia repair $p<0.001)$. Correlation and consistency between IPQ and sf-IPQ scores using ICC was 0.78 (95\% confidence interval $0.73-0.82, p<0.001)$. McNemar's test showed a difference between IPQ and sf-IPQ, with a higher proportion of patients attaining a score indicating substantial pain on the sf-IPQ ( $p=0.013)$.

The distribution of SF-MPQ scores differed between patients rating an sf-IPQ score $<3$ and those rating an sfIPQ score $\geq 3$, as seen in Fig. 4 .

\section{Discussion}

The main conclusion from this study is that the sf-IPQ is a reliable disease-specific instrument for the assessment of groin pain and is well suited to be used, in place of the IPQ, in daily clinical practice. The advantage of the sf-IPQ is its simplified layout and condensed form. The sf-IPQ is a onepage document which takes less than 1 min to complete, whereas the original IPQ is a four-page questionnaire and takes approximately $10 \mathrm{~min}$ to complete. The brevity of the sf-IPQ decreases the risk of patients choosing not to complete the questionnaire. The statistical analyses confirm that the sf-IPQ provides comparable information to that of the original IPQ. The analyses also show that the sf-IPQ produces a slightly higher pain score than the IPQ, indicating that the sf-IPQ is a more sensitive instrument for measuring inguinal pain. This could be considered a 


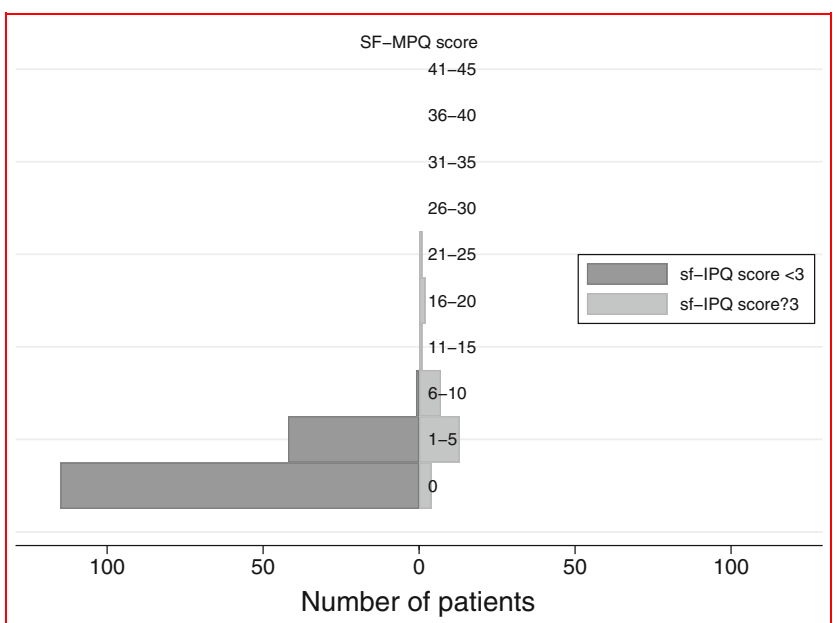

Fig. 4 SF-MPQ scores compared with sf-IPQ scores for 186 patients 3 years after inguinal hernia repair, answers grouped by sf-IPQ score ( $<3$ vs. $\geq 3$ )

desirable trait in an instrument intended to direct awareness of the healthcare provider towards patients who are likely to benefit from a more thorough follow-up and further consultation.

A crucial prerequisite for evaluating strategies to manage persisting pain after hernia surgery is a reliable instrument to evaluate inguinal pain. Such an instrument must be user-friendly to ensure a high response rate. Because of difficulties in defining the subjective experience of pain, instruments for its assessment are prone to be too extensive. This increases the risk of it becoming too laborious for the patient to answer, leading to inadequate response rates in settings where it is not feasible to allocate specific resources dedicated to ensure (near) complete response rates.

There are several general instruments available to evaluate pain regardless of origin. One of these is the McGill pain questionnaire which was used as the reference pain instrument in this study [5]. The original IPQ is not the only disease-specific instrument available for evaluating post-operative inguinal pain. Carolinas Comfort Scale (CCS) is a validated, disease-specific, well-established questionnaire mainly focusing on the outcome quality of life [16]. The CCS is trademarked and requires a license agreement to be used. The sf-IPQ, on the other hand, focuses on pain as outcome after hernia surgery and is free for anyone to use.

The Core Outcome Measure Index adapted for patients with hernia (COMI-hernia) is a multidimensional, patientorientated outcome questionnaire [17]. An overall COMI score is generated from the average score of answers to questions on various dimensions. The COMI-hernia instrument includes several modalities and may be considered too extensive for routine clinical use, rather like the original IPQ. Multidimensional questionnaires provide information on several outcome measures after surgery. Aggregation of this information into a single score may seem convenient, but this actually makes it more difficult to interpret data since the score combines evaluations of different issues. The sf-IPQ is restricted to the dimension of pain, thus making the information gained easier to interpret.

Statistical analyses showed agreement between the sfIPQ and the IPQ. When calculated with Cohen's kappa, the agreement is "substantial" according to Landis and Koch's guidelines [18]. The correlation and consistency of the sfIPQ and the IPQ using the ICC was at an "excellent" level according to the guidelines stipulated by Cicchetti et al. [19]. The sf-IPQ may be considered comparable to and interchangeable with the IPQ and may thus be recommended as an alternative instrument for the measurement of post-operative pain.

Despite the significant agreement, correlation and consistency between the sf-IPQ and IPQ scores, McNemar's test showed consistently slightly higher scores using the sfIPQ. This could be considered an advantage of this instrument as discussed above. There is a minor difference in layout between the two forms, which could have been a reason for the difference in scores. When answering the IPQ, if respondents state they have not experienced pain the previous week, they are instructed to ignore the set of questions regarding limitation of activities due to inguinal pain. On the other hand, when answering the sf-IPQ, if no pain has been experienced the past week, respondents are not explicitly instructed to refrain from answering questions in Item 2 (regarding activities limited by pain). However, when analysing answer patterns, the difference in layout did not explain the slightly higher score using the sf-IPQ.

Non-responders were younger than responders, but apart from this there were no differences between the groups, which would indicate that the results also apply to larger populations.

The number of responders after two reminders did not reach that required according to the power calculation, although the response rate of $69.8 \%$ is considered fully acceptable.

sf-IPQ scores were compared to the SF-MPQ scores (a well-established pain instrument) to evaluate pain score distribution. This revealed that patients with higher sf-IPQ scores also scored higher with the SF-MPQ, i.e. both questionnaires measure the same domain (pain). Whereas the SF-MPQ registers general pain, the sf-IPQ is more accurate with respect to groin pain associated with inguinal hernia surgery. 
The main difference between the sf-IPQ and the IPQ is the complexity of the questionnaires. The advantage of the sf-IPQ compared to other existing instruments is its compact design and its user-friendliness. The sf-IPQ may not include all the information required for detailed research, but we found it to be a simple disease-specific pain instrument appropriate for daily clinical use, providing concise outcome information entirely comparable with that from the original IPQ.

Acknowledgements This study was supported with grants from Stockholm County Council and the Swedish Society of Medicine. The authors would like to acknowledge Hans Järnbert Pettersson, Ph.D., Karolinska Institutet, for his important contribution to the statistical planning.

Open Access This article is distributed under the terms of the Creative Commons Attribution 4.0 International License (http://crea tivecommons.org/licenses/by/4.0/), which permits unrestricted use, distribution, and reproduction in any medium, provided you give appropriate credit to the original author(s) and the source, provide a link to the Creative Commons license, and indicate if changes were made.

Funding Grants from Stockholm County Council ALF Project Funding (ALF 20160009) and the Swedish Society of Medicine (SLS694141).

\section{References}

1. Bay-Nielsen M, Perkins FM, Kehlet H, Danish HD (2001) Pain and functional impairment 1 year after inguinal herniorrhaphy: a nationwide questionnaire study. Ann Surg 233:1-7

2. Courtney CA, Duffy K, Serpell MG, O'Dwyer PJ (2002) Outcome of patients with severe chronic pain following repair of groin hernia. Br J Surg 89:1310-1314

3. Magnusson N, Gunnarsson U, Nordin P, Smedberg S, Hedberg M, Sandblom G (2015) Reoperation for persistent pain after groin hernia surgery: a population-based study. Hernia 19:45-51
4. Treede RD, Rief W, Barke A, Aziz Q, Bennett MI, Benoliel R et al (2015) A classification of chronic pain for ICD-11. Pain 156:1003-1007

5. Melzack R (1975) The McGill Pain Questionnaire: major properties and scoring methods. Pain 1:277-299

6. Kehlet H, Jensen TS, Woolf CJ (2006) Persistent postsurgical pain: risk factors and prevention. Lancet 367:1618-1625

7. Nienhuijs S, Staal E, Strobbe L, Rosman C, Groenewoud H, Bleichrodt R (2007) Chronic pain after mesh repair of inguinal hernia: a systematic review. Am J Surg 194:394-400

8. Franneby U, Sandblom G, Nordin P, Nyren O, Gunnarsson U (2006) Risk factors for long-term pain after hernia surgery. Ann Surg 244:212-219

9. Olsson A, Sandblom G, Franneby U, Sonden A, Gunnarsson U, Dahlstrand U (2017) Impact of postoperative complications on the risk for chronic groin pain after open inguinal hernia repair. Surgery 161:509-516

10. Franneby U, Gunnarsson U, Andersson M, Heuman R, Nordin P, Nyren O et al (2008) Validation of an Inguinal Pain Questionnaire for assessment of chronic pain after groin hernia repair. Br J Surg 95:488-493

11. Cleeland CS, Ryan KM (1994) Pain assessment: global use of the Brief Pain Inventory. Ann Acad Med Singap 23:129-138

12. Swedish Hernia Register (2017)

13. Melzack R (1987) The short-form McGill Pain Questionnaire. Pain 30:191-197

14. Burckhardt CS, Bjelle A (1994) A Swedish version of the shortform McGill Pain Questionnaire. Scand J Rheumatol 23:77-81

15. Shrout PE, Fleiss JL (1979) Intraclass correlations: uses in assessing rater reliability. Psychol Bull 86:420-428

16. Heniford BT, Lincourt AE, Walters AL, Colavita PD, Belyansky I, Kercher KW et al (2018) Carolinas comfort scale as a measure of hernia repair quality of life: a reappraisal utilizing 3788 international patients. Ann Surg 267(1):171-176

17. Staerkle RF, Villiger $P$ (2011) Simple questionnaire for assessing core outcomes in inguinal hernia repair. Br J Surg 98:148-155

18. Landis JR, Koch GG (1977) The measurement of observer agreement for categorical data. Biometrics 33:159-174

19. Cicchetti DV (1994) Guidelines, criteria, and rules of thumb for evaluating normed and standardized assessment instruments in psychology. Psychol Assess 6:284-290 